\title{
A protective stent coating
}

Stents are implanted to dilate arteries narrowed by atherosclerotic plaques and to revascularize coronary arteries following myocardial infarction. However, in up to $20 \%$ of patients the regrowth of cells in the inner vessel wall (neointima) or incomplete healing of the endothelial cell lining in the vessel lumen can cause recurrent arterial obstruction - also known as restenosis. Drug-eluting stents, which slowly release anti-proliferative agents, significantly reduce the rate of restenosis compared with bare-metal stents but they are associated with an increased risk of late-stent thrombosis. Soehnlein et al. now show that coating stents with an antimicrobial peptide released from neutrophils - cathelicidin - could represent a novel strategy for preventing restenosis.

Arterial injury resulting from stent implantation is known to induce an inflammatory response that involves the recruitment of neutrophils. At damaged sites, neutrophils secrete various proteins that activate both circulating and resident cells. First, the authors showed that neutrophil depletion increased neointima formation in atherosclerosis-prone apolipoprotein E-deficient $\left(\right.$ Apoe $\left.^{-/-}\right)$ mice, indicating a beneficial effect of neutrophils. Moreover, they found that the neutrophil-released, positively charged polypeptide cathelicidin, known in mice as cathelin-related antimicrobial peptide (CRAMP), is a key regulator in limiting neointima formation. CRAMP was deposited by neutrophils at sites of endothelial cell injury, and the phenotype of neutropaenic $A_{p o e^{-/-}}$mice strongly resembled that of $A$ poe $e^{-/-}$mice transplanted with $\mathrm{Cramp}^{-/-}$bone marrow.

The authors found that in the blood vessel lumen, CRAMP recruits endothelial progenitor cells to sites of arterial injury, and that this effect is mediated by formyl peptide receptor 2 (FPR2). Furthermore, they showed that the interaction of human cathelicidin (LL-37) with FPR2 on endothelial progenitor cells promotes endothelial progenitor cell survival under inflammatory
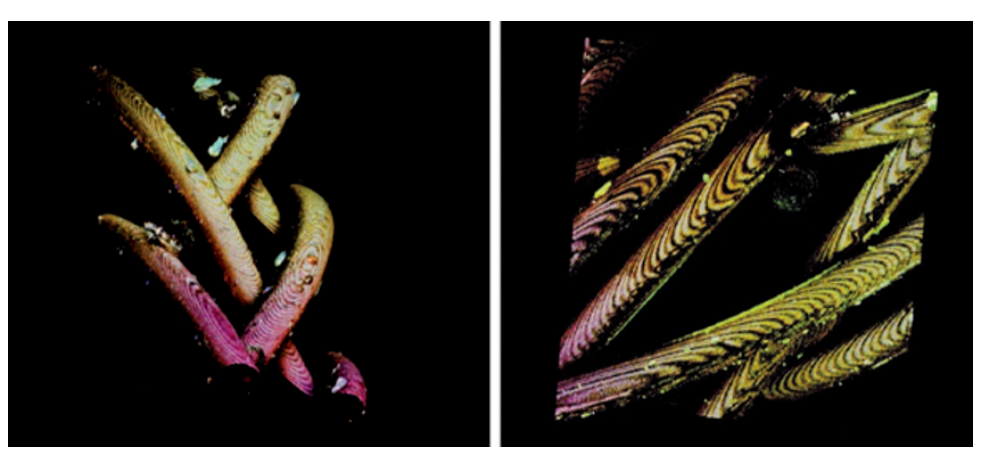

Bare-metal stent (left) and cathelicidin-coated stent (right): images courtesy of O. Soehnlein, Ludwig-Maximilians University of Munich, Germany. conditions and accelerates re-endothelialization by stimulating the release of pro-angiogenic factors.

Importantly, when Soehnlein et al. implanted LL-37-coated stents into a mouse model of atherosclerosis they found a decreased incidence of restenosis. This paper not only sheds light on the mechanisms through which neutrophils promote re-endothelialization and reduce the extent of neointima formation in arterial disease, but also highlights the possibility of translating these findings into the clinic, as cathelicidin-coated stents could help prevent restenosis. Monica Hoyos Flight

ORIGINAL RESEARCH PAPER Soehnlein, O. et al. Neutrophil-derived cathelicidin protects from neointimal hyperplasia. Sci. Transl. Med. 3 103ra98 (2011) 